The oral kinase inhibitors show considerable promise for extending the survival of patients with RCC. Motzer et al. recently presented results that favor sunitinib, from their phase III trial of interferon a versus sunitinib as first-line therapy for metastatic RCC, at the American Society of Clinical Oncology June 2006 plenary session.

Original articles Motzer RJ et al. (2006) Sunitinib in patients with metastatic renal cell carcinoma. JAMA 295: 2516-2524 Ratain MJ et al. Phase II placebo-controlled randomized discontinuation trial of sorafenib in patients with metastatic renal cell carcinoma. J Clin Oncol 24: 2505-2512

\section{Accuracy of depression evaluation in hemodialysis patients}

Patients with end-stage renal disease on chronic hemodialysis often experience symptoms of depression. Adverse effects of dialysis including sleep disturbance, fatigue, and appetite and weight changes, however, could lead to erroneous diagnoses of depression as measured by self-report depression scales. Hedayati et al. explored the prevalence of depression in a group of 98 patients on dialysis and assessed the accuracy of different self-report scales.

The physician-administered Structured Clinical Interview for Depression (SCID) scale indicated that $26.7 \%$ of the group had a depressive disorder, and $17.3 \%$ major depression. In comparison with this scale, the Beck Depression Inventory (BDI), Center for Epidemiological Study of Depression (CESD) and Cognitive Depression Inventory (CDI) self-report scales were poor diagnostic tools. The BDI and CESD performed better, however, when their cutoff points for a diagnosis of depression were raised beyond those validated in the general population-supporting the notion that self-report scales record additional somatic depressive symptoms in hemodialysis patients that are not true indicators of depression. In further analyses, the CESD was found to be more accurate than the BDI at identifying individuals with depression.

The authors conclude that at the proposed new cutoffs the BDI and CESD are sufficiently accurate to be used as screening tools; they suggest that these tools be used to evaluate dialysis patients for depression at approximately yearly intervals, with patients with positive results being formally assessed by a physician or referred for psychiatric assessment. They do not recommend the CDI for screening because of its low sensitivity.

Original article Hedayati SS et al. (2006) The predictive value of self-report scales compared with physician diagnosis of depression in hemodialysis patients. Kidney Int 69: 1662-1668

\section{Perineural invasion is not prognostic in prostate cancer treated with brachytherapy}

The importance of perineural invasion (PNI) as a prognostic indicator in patients with prostate cancer is controversial and the data on the prognostic significance of PNI in those patients receiving brachytherapy are sparse. Researchers from the Cleveland Clinic Foundation, $\mathrm{OH}$, USA have now reported that PNI does not predict biochemical failure in patients with prostate cancer treated with brachytherapy.

In this matched-pair analysis, all patients received brachytherapy of 144 Gy to the prostate. Cases (biochemical failure) were matched with controls (no biochemical failure) for clinical stage, Gleason score, prostate-specific antigen level, age, race, and androgen deprivation status. A total of 56 pairs were available for analysis. Median follow-up time between cases and controls was comparable (58.5 and 53.5 months, respectively). The number of patients with PNI did not differ significantly between the two groups. Although there was a slight trend towards increased biochemical failure in patients with PNI, Kaplan-Meier analysis revealed that the difference between PNI-positive and PNI-negative patients was not statistically significant $(P=0.4014)$.

Although this study was limited by sample size, the authors conclude that the data are consistent with other reports in the literature that PNI does not have prognostic value in patients with prostate cancer. They suggest that post-therapy external beam radiation for patients with $\mathrm{PNI}$ is, therefore, unnecessary.

Original article Weight CJ et al. (2006) Perineural invasion on prostate needle biopsy does not predict biochemical failure following brachytherapy for prostate cancer. Int $J$ Radiat Oncol Biol Phys 65: 347-350 